\title{
Therapeutic plasma exchange for neuromyelitis optica spectrum disorder: A multicenter retrospective study by the ASFA neurologic diseases subcommittee
}

\author{
Tina S. Ipe 1,2 (i) I Jay S. Raval $^{3,4}$ | Leonor P. Fernando ${ }^{5}$ | Amit Gokhale ${ }^{6}$ | \\ Cyril Jacquot $^{7}$ | Andrew D. Johnson ${ }^{8}$ | Haewon C. Kim ${ }^{9}$ | Grace F. Monis ${ }^{5}$ | \\ Yunchun D. Mo $^{7}$ | Shanna M. Morgan ${ }^{10}$ | Monica B. Pagano ${ }^{11}$ | Huy P. Pham ${ }^{12}$ | \\ Kimberly Sanford ${ }^{13}$ | Amy E. Schmidt ${ }^{14}$ | Joseph Schwartz ${ }^{15}$ | Amy Waldman' |

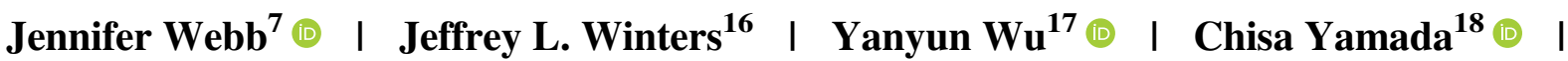

Edward C. C. Wong ${ }^{19,20}$

\footnotetext{
${ }^{1}$ Department of Pathology and Laboratory Medicine, University of Arkansas for Medical Sciences, Little Rock, Arkansas

${ }^{2}$ Department of Pathology and Genomic Medicine, Houston Methodist Hospital, Houston, Texas

${ }^{3}$ Department of Pathology, University of New Mexico, Albuquerque, New Mexico

${ }^{4}$ Department of Pathology and Laboratory Medicine, University of North Carolina, Chapel Hill, North Carolina

${ }^{5}$ Department of Pathology and Laboratory Medicine, University of California, Davis, Sacramento, California

${ }^{6}$ Department of Pathology, Stony Brook University, Stony Brook, New York

${ }^{7}$ Department of Laboratory Medicine and Hematology, Children's National Health System, Washington, District of Columbia

${ }^{8}$ Department of Laboratory Medicine and Pathology, University of Minnesota, Minneapolis, Minnesota

${ }^{9}$ Department of Pediatrics and Pathology, Children's Hospital of Philadelphia, Philadelphia, Pennsylvania

${ }^{10}$ American Red Cross, Minneapolis, Minnesota

${ }^{11}$ Department of Laboratory Medicine, University of Washington, Seattle, Washington

${ }^{12}$ Department of Pathology, University of Southern California, Los Angeles, California

${ }^{13}$ Department of Pathology, Virginia Commonwealth University, Richmond, Virginia

${ }^{14}$ Department of Pathology and Laboratory Medicine, University of Rochester Medical Center, Rochester, New York

${ }^{15}$ Department of Pathology and Cell Biology, Columbia University, New York, New York

${ }^{16}$ Department of Laboratory Medicine and Pathology, Mayo Clinic, Rochester, Minnesota

${ }^{17}$ Bloodworks Northwest, Seattle, Washington

${ }^{18}$ Department of Pathology, University of Michigan, Ann Arbor, Michigan

${ }^{19}$ Department of Coagulation, Quest Diagnostics Nichols Institute, Chantilly, Virginia

${ }^{20}$ Department of Pediatrics and Pathology, George Washington School of Medicine and Health Sciences and Children's National Hospital, Washington, District of Columbia
}

\section{Correspondence}

Tina S. Ipe, 4301 W. Markham St., Slot

503, Little Rock, AR 72205.

Email: tipe@uams.edu

\begin{abstract}
Importance: Neuromyelitis optica/neuromyelitis optica spectrum disorder patients' response to therapeutic plasma exchange (TPE) is currently incompletely characterized. Objective: Our study aims to understand the clinical status improvement of neuromyelitis optica/neuromyelitis optica spectrum disorder patients treated with TPE.
\end{abstract}

Tina S. Ipe and Jay S. Raval contributed equally to this study and are co-first authors. 
Design, Setting, and Participants: This is a multicenter retrospective study conducted between 1 January 2003 and 31 July 2017 at 13 US hospitals performing apheresis procedures. Subjects studied were diagnosed with neuromyelitis optica/ neuromyelitis optica spectrum disorder who received TPE during presentation with acute disease.

Main Outcomes and Measures: The primary outcome was clinical status improvement in patients treated with TPE. Secondary measures were procedural and patient characteristics associated with response to treatment.

Results: We evaluated 114 patients from 13 institutions. There was a female predilection. The largest ethnic group affected was non-Hispanic Caucasian. The average age of diagnosis was 43.1 years. The average time to diagnosis was 3.1 years. On average, five procedures were performed during each treatment series. The most commonly performed plasma volume exchange was 1.0 to 1.25 using $5 \%$ albumin as replacement fluid. Most patients (52\%) did not require an additional course of TPE and noted "mild" to "moderate" clinical status improvement. Maximal symptom improvement appeared by the fourth or fifth TPE treatment.

Conclusion and Relevance: TPE improved the clinical status of patients. Adults responded more favorably than children. Procedural characteristics, including number of TPEs, plasma volume exchanged, and replacement fluid used, were similar between institutions. TPE was well-tolerated and had a low severe adverse event profile.

\section{K E Y W O R D S}

clinical status improvement, demographics, procedural, registry

\section{1 | INTRODUCTION}

Neuromyelitis optica (NMO), previously known as Devic's disease, is an inflammatory disorder of the central nervous system characterized by immune-mediated demyelination and axonal damage predominantly targeting the optic nerves and spinal cord. NMO was initially thought to be a variant of multiple sclerosis (MS), with variable expression of MS symptoms. However, with increased knowledge of NMO pathogenesis, imaging features, biomarkers, and treatment response, NMO is now considered a distinct neurologic condition from MS. Although the exact cause of NMO is still unknown, it is believed that a NMO-specific immunoglobulin $\mathrm{G}(\mathrm{IgG})$ autoantibody, aquaporin 4 (AQP4), plays a role in NMO pathogenesis. ${ }^{1}$ The AQP4 antibody was identified in 2004; it localizes at the blood-brain barrier and has a sensitivity of $73 \%$ to $91 \%$ and specificity of $91 \%$ to $100 \%$ as a diagnostic marker for NMO. ${ }^{1,2}$ The AQP4 antigen is a water channel protein that is highly concentrated in spinal cord gray matter and at the astrocytic foot processes in the blood-brain barrier. Anti-AQP4 is pathognomonic for NMO and helps to distinguish NMO patients from those with MS. Antibody status was included in the revised
NMO diagnostic criteria in $2006 .{ }^{3}$ Furthermore, the addition of this autoantibody created a broader spectrum to define this disease, known as NMO spectrum disorders (NMOSD). In addition to serving as a diagnostic disease marker, anti-AQP4 IgG levels in the serum have been shown to correlate with disease activity as their levels decrease after immunosuppressive therapy and during remissions. ${ }^{2}$ Higher titers of this antibody were found in patients with severe clinical manifestations of the disease, such as blindness or lesions involving $\geq 3$ vertebral segments of the spinal cord. ${ }^{2}$

The prevalence of NMO/NMOSD in the United States is approximately 4000 to 8000 patients. ${ }^{4}$ It is estimated that $1 \%$ to $2 \%$ of patients diagnosed with MS actually have NMO/NMOSD. NMO/NMOSD occurs more commonly in females (up to 4-5 times as frequently than in males) and in patients of Caucasian descent. ${ }^{4} \mathrm{NMO} / \mathrm{NMOSD}$ patients typically have an older age of onset compared to patients with multiple sclerosis. ${ }^{4}$ NMOSD is typically sporadic, although a few familial cases have been documented. ${ }^{5}$ The typical clinical features of NMO are acute and include varying degrees of vision loss (optic neuritis) and features of transverse myelitis, which can include limb weakness, sensory 
loss, and bladder dysfunction. Hypothalamic and brainstem involvement occurs in a minority of patients, which can manifest as hiccups, intractable nausea, and respiratory failure. ${ }^{6} \mathrm{NMO} / \mathrm{NMOSD}$ typically has a relapsing course.

The treatment for acute and chronic episodes of NMO/NMOSD include glucocorticosteroids and therapeutic plasma exchange (TPE). ${ }^{7}$ According to the American Society for Apheresis (ASFA) Special Issue, TPE is a Category II (second line) treatment for acute episodes of NMO/NMOSD. ${ }^{8} \mathrm{TPE}$ has been shown to be beneficial in the management of acute and chronic episodes of NMO/NMOSD, likely through the removal of the AQP4 antibody and other inflammatory substances from the blood. ${ }^{9}$ A study of patients suffering from acute NMO/NMOSD attacks demonstrated that early initiation of TPE along with intravenous glucocorticoids resulted in greater patient clinical improvement compared with the delayed introduction of TPE following steroid therapy. ${ }^{10}$ Although observational studies show TPE is beneficial in NMO/NMOSD, limited specific information is known regarding these apheresis procedures, patients' degree of response to these procedures, and the characteristics of patients who benefited with a positive response. As part of the neurologic diseases subcommittee of the ASFA research committee, we conducted a multi-institutional retrospective study to help answer these questions. The purpose of this study was to gain an understanding of specific TPE procedural information, response of acute NMO/NMOSD symptoms to TPE, and patient characteristics associated with TPE response. In this study, we determine the safety and efficacy of TPE in the treatment of NMO/NMOSD.

\section{2 | MATERIALS AND METHODS}

\section{1 | Subjects}

We conducted a retrospective medical chart review of patients with a diagnosis of NMO/NMOSD confirmed by review of their medical chart at the initiation of treatment performed by apheresis physicians at their respective institutions. TPE series reviewed occurred over 15 years from January 1, 2003 to July 31,2017 . Because our input of data into this retrospective began in 2010, patients were included in the study if they met either the NMO or NMOSD diagnostic criteria proposed in 2006 or 2015 , respectively. The 2006 criteria requires patients to have optic neuritis and transverse myelitis plus two of the following clinical parameters: (a) contiguous spinal cord lesions extending three or more vertebral segments, (b) nondiagnostic magnetic resonance imaging (MRI) of the brain with normal findings or with findings not consistent with MS, and/or (c) NMO IgG seropositivity. ${ }^{11}$ The 2015 criteria require a positive test for AQP4-IgG, exclusion of alternative diagnoses, and one of the following core clinical characteristics: (a) optic neuritis, (b) acute myelitis, (c) area postrema syndrome, (d) acute brainstem syndrome, (e) symptomatic narcolepsy or acute diencephalic clinical syndrome with NMOSD-typical MRI lesions, or (f) symptomatic cerebral syndrome with NMOSD-typical brain lesions. ${ }^{12}$ Diagnostic criteria for seronegative or unknown AQP4-IgG status require meeting additional criteria such as acute optic neuritis, acute myelitis, area postrema syndrome, and acute brain stem syndrome. ${ }^{12}$ All institutions that participated in the study had approval from their institutional review board (IRB) and had a current data use agreement (DUA) with the ASFA. Patients with confirmed NMO/NMOSD were included in a relational database (REDCap). We defined pediatric patients as those $\leq 21$ years old.

\section{2 | Terms}

Patients with new-onset clinical symptomology of NMOSD for at least 24 hours were defined as having an acute disease. TPE series involved a single treatment course with a maximum of 10 consecutive procedures. Distinct TPE series, defined as those separated by $>2$ weeks, were labeled as unique TPE treatment arms in this study. The clinical status of patients was determined by reviewing notes from the patient's neurologist and apheresis practitioners to demonstrate if an improvement was seen during the apheresis treatment course. Clinical status was assessed on a scale from 1 to 5: (1) no improvement, (2) mild improvement (slight but definite change and with no impact on the daily function), (3) moderate improvement (an obvious improvement that impacts daily function), (4) marked improvement (a significant difference from pre-TPE baseline with major functional daily improvement), and (5) full recovery from pre-first TPE baseline.

\section{3 | Registry}

REDCap is a relational data capture tool created by Vanderbilt University for clinical and translational research. Our registry was used to collect detailed, de-identified patient demographic, and NMOSD clinical information, and TPE information including schedule and adverse events. We validated the data with an independently conducted two-person review of the information entered into the registry by individuals at the same institution. The time of data entry varied, with the first entries submitted in 2010 .

\section{4 | Statistical analysis}

The primary outcome measured was clinical status improvement (CSI). CSI was analyzed in relation to patient characteristics and TPE. No improvement was defined as a CSI score of 1 to 2 and improvement was defined as a score of 3 to 5 . Summary statistics were assessed using mean, SD, 
and count frequency. Longitudinal regression was performed using XTREG with Stata (V15, College Station, Texas) to model mean change in clinical status from baseline. Chisquare contingency testing was also performed to determine the association between NMO IgG seropositivity and time to treatment, relapse, and CSI. Statistical significance was defined as $P<.05$.

\section{3 | RESULTS}

\section{1 | Patient demographics}

A total of 114 patients undergoing 148 treatment series (TS) from 13 sites-two pediatric $(15.4 \%, 2 / 13)$ and 11 adult or adult/pediatric $(84.6 \%, 11 / 13)$ apheresis centers-were evaluated. All census regions within the United States were represented. The 13 institutions that participated in the study were: Houston Methodist Hospital, University of North Carolina, University of California Davis Medical Center, Yale School of Medicine, Children's National Health System, Virginia Commonwealth University, University of Rochester Medical Center, University of Washington/ Bloodworks NW, Children's Hospital of Philadelphia, Columbia University, Mayo Clinic, University of Minnesota, and University of Michigan. The two groups with the largest patient ethnicities included non-Hispanic Caucasian $(49.1 \%, 56 / 114)$ and African American ( $\mathrm{N}=28.0 \%, 32 / 114)$ (Figure 1A). Patients were predominantly female $(81.6 \%, 93 / 114)$, with a female to male ratio of 4.4:1 (Figure 1B). The average age at diagnosis was 43.1 years \pm 19.4 (range $0.7-84$ years)

\section{2 | Patient clinical characteristics}

The core clinical characteristics exhibited by patients in their TS in this study were ranked from highest to lowest in frequency: transverse myelitis $(74.3 \%, 110 / 148)$, spinal cord MRI lesion extending over three or more contiguous vertebral segments $(60.8 \%, 90 / 148)$, NMO IgG seropositivity $(59.5 \%, 88 / 148)$, optic neuritis $(41.2 \%, 61 / 148)$, and normal brain MRI or non-specific white matter lesions $(40.5 \%$, 60/148) (Figure 2A). The most common clinical findings present in patients before the initiation of TPE were paraparesis $(62.8 \%, 93 / 148)$, bilateral sensory loss (53.4\%, 79/148), blindness $(44.6 \%, 66 / 148)$, and sphincter dysfunction $(37.2 \%, 55 / 148)$.

\section{3 | Procedural characteristics}

A total of 811 TPE procedures associated with 148 TS were analyzed. TPE was performed every other day, not including weekends, at most institutions $(60.8 \%, 90 / 148)$ with a median and mode of five exchanges over 10 days (range of

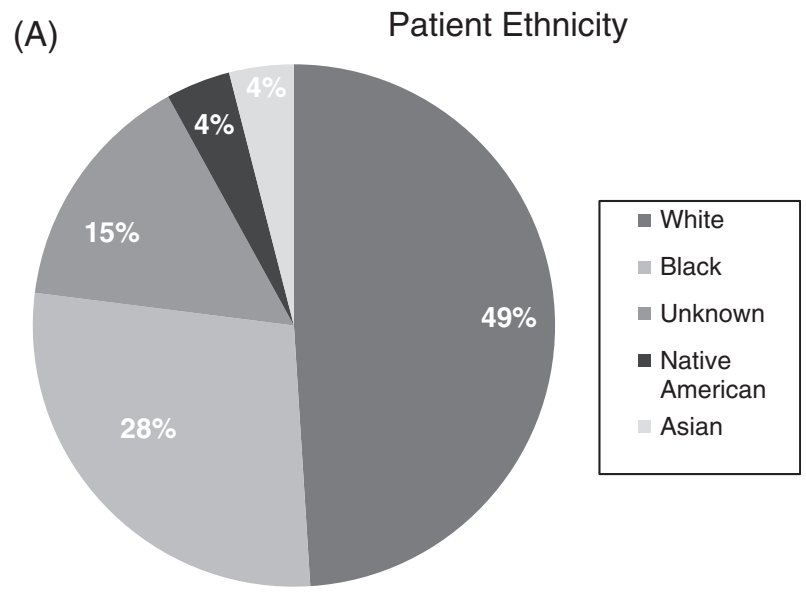

(B)

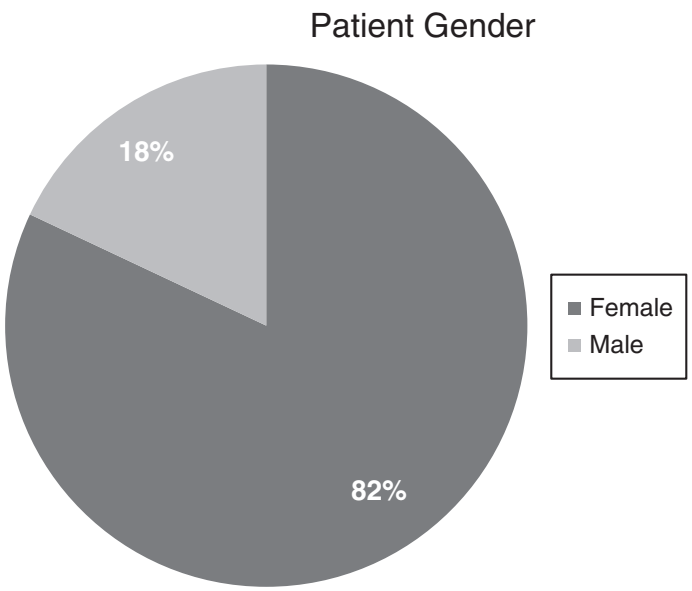

F I G URE 1 Demographics of the patient population used in the study: A, patient ethnicity and B, patient gender

2-10 procedures per TS). The most commonly performed plasma volume exchange was 1.0 to $1.25(89.9 \%, 133 / 148)$, and 5\% albumin was the predominantly used replacement fluid $(99.3 \%, 797 / 803)$. Most procedures utilized a fluid balance of $100 \%$ (97.9\%, 786/803). Anticoagulant Citrate Dextrose Solution A (ACD-A) was the most commonly used anticoagulant $(91.0 \%, 728 / 800)$. Most procedural encounters were performed using a central venous catheter with internal jugular venous access $(82.5 \%, 659 / 799)$. Other identified access sites included subclavian central venous catheter (5.4\%, 43/799), peripheral venous access $(4.9 \%, 39 / 799)$, and femoral line $(3.9 \%, 31 / 799)$. While TPE was welltolerated by most patients, a minority of procedures were associated with an adverse event $(9.9 \%, 75 / 759)$. The most commonly specified adverse event was citrate toxicity $(3.6 \%, 27 / 759)$. Other various infrequent adverse events $(6.3 \%, 48 / 759)$ included venous access difficulties, hypotension, and allergic reactions. Only one severe adverse event was noted, which was a severe allergic/anaphylactic reaction to 5\% albumin; this patient had their remaining TPE procedures performed with a combination of $0.9 \%$ normal saline and hetastarch as replacement fluid. Average time from 
F IG URE 2 Patient diagnostic criteria and symptoms at baseline. Neuromyelitis optica/ neuromyelitis optica spectrum disorder (NMO/NMOSD) diagnostic criteria

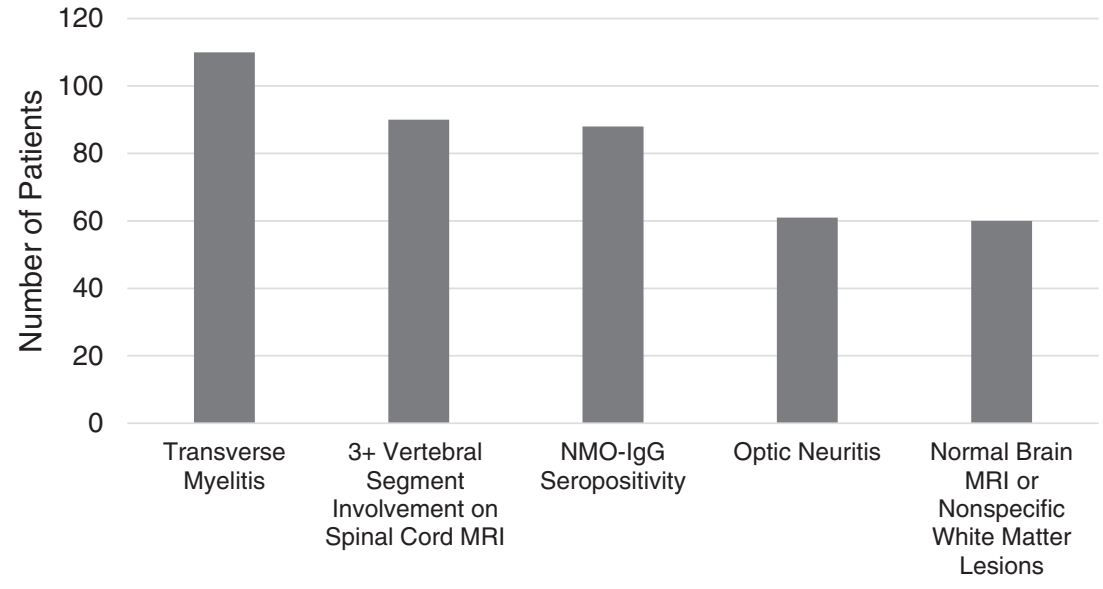

Patient Diagnostic Criteria diagnosis to treatment with TPE was 22.8 months \pm 46.8 (range 0 to 276 months).

\subsection{Efficacy of TPE in acute NMO/NMOSD}

Regarding clinical outcome, both adults and children showed CSI over the course of TPE using multilevel-ordered logistic modeling (Figure 3A). In comparison to children, adults had better improvement over the course of treatment, with continued symptom improvement through the 10th procedure. Overall, children had a 1.59 lower CSI score than adults $(P<.001)$. There was a significant difference over time in both groups $(P<.001)$. There was also a non-significant trend of those between the ages of 22 and 50 years-of-age toward better CSI (Figure 3B). Most patients $(52 \%, 59 / 114)$ did not require an additional series of TPE and noted "mild" to "moderate" CSI after one series of TPE. Maximal sign/symptom improvement appeared to be achieved by the fourth or fifth TPE treatment. Disease relapse requiring TPE treatment was not associated with the presence of AQP4 receptor antibody (Figure 4A). Similarly, having the AQP4 antibody was not associated with CSI (Figure 4B). Time to treatment after initial diagnosis did not affect patient clinical response to TPE (Figure 4C).

\section{4 | DISCUSSION}

Analysis of the NMO/NMOSD registry data demonstrated that treatment of acute episodes with TPE was feasible, tolerable, and had a low severe adverse event profile. Patients diagnosed with NMO or NMOSD according to the diagnosis criteria proposed in 2006 and 2015 who were treated with TPE during the acute phase of their disease were included in this retrospective study. This study showed a female and Caucasian predisposition to the disease, similar to an epidemiological study performed in the United States by Mealy et al. $^{4}$ Our patients were slightly older (43.1 years) at
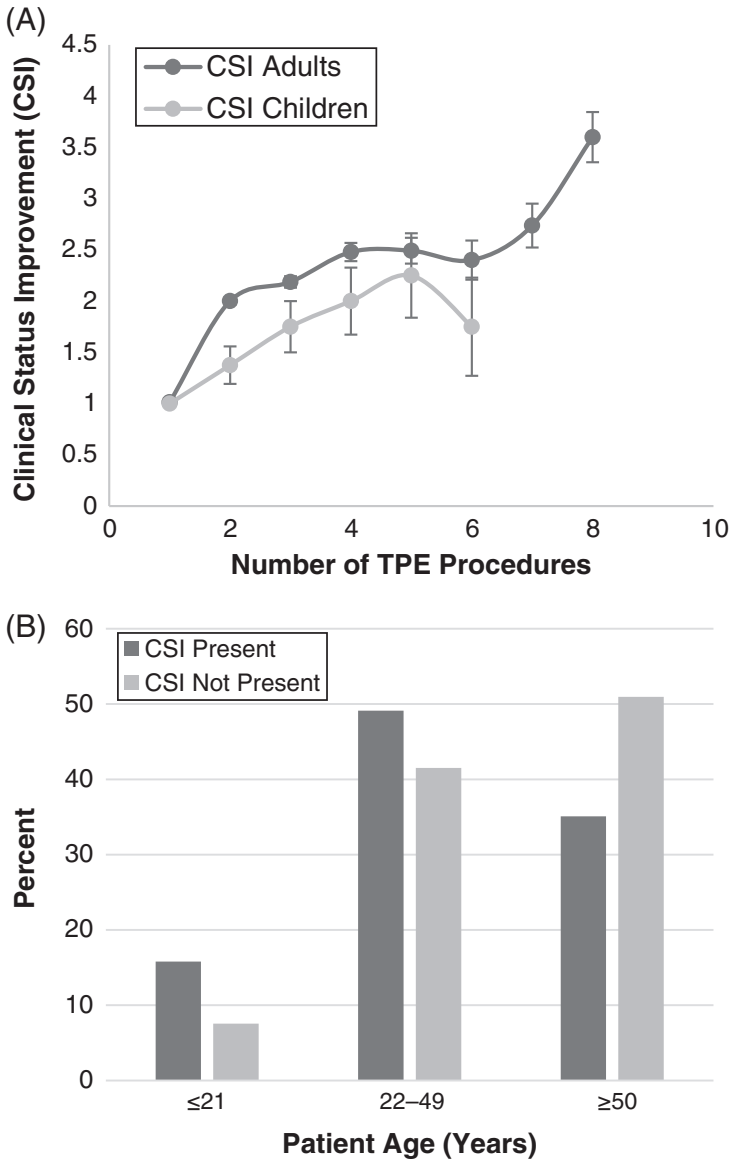

F I G U RE 3 A, Clinical symptomology improvement (CSI) grouped by patient age. B, Symptoms observed at baseline therapeutic plasma exchange series grouped by clinical symptomology improvement (CSI)

presentation than those in the previous study (41.1 years). Most patients $(>50 \%)$ presented with the core clinical findings of transverse myelitis and optic neuritis, as well as other parameters including contiguous spinal cord lesions, nonMS brain imaging, and AQ4-IgG seropositivity. These findings are consistent with other studies. ${ }^{1,4}$ 

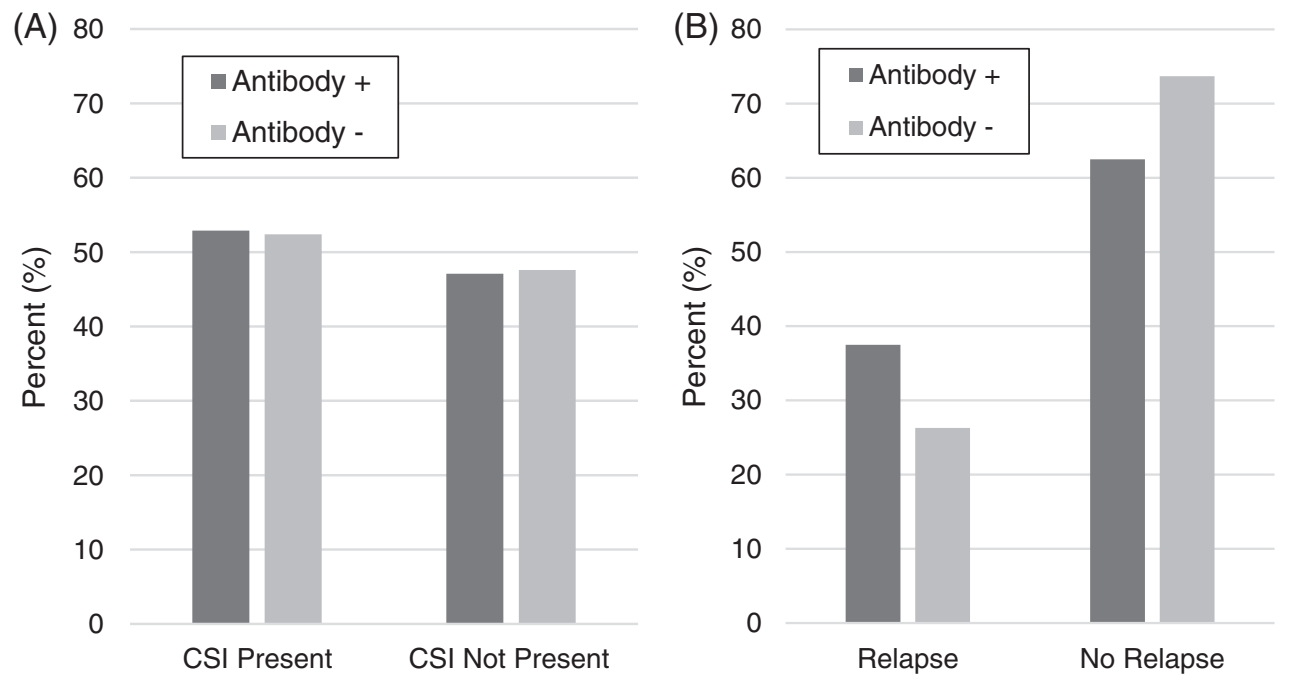

F I G URE 4 Qualitative serum anti-AQP4 was not significantly associated with any of the following studied outcomes as determined by Fisher's exact test: A, clinical symptomology improvement (CSI) $(P=1.0)$; B, disease relapse $(P=.57)$; or $\mathrm{C}$, lag time to treatment $(P=.11)$

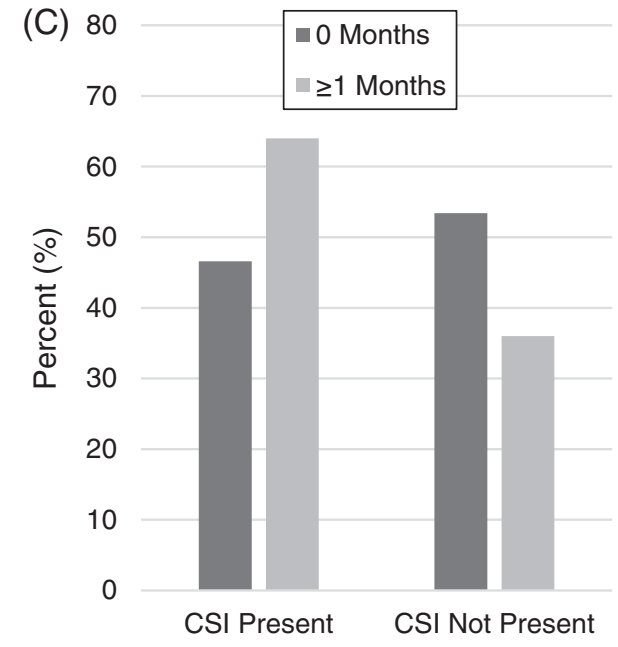

TPE has been shown to be efficacious in the treatment of central nervous system inflammatory disorders, including NMO/NMOSD, in small, randomized controlled trials and observational studies. ${ }^{7,13-18}$ Its proposed mechanism of action includes the removal of autoantibodies, cytokines, and inflammatory markers. ${ }^{9}$ TPE was performed similarly at the institutions that participated in this study. Specifically, at the institutions participating in this study, TPE was used as adjuvant therapy or after patients had failed other immunomodulatory treatments, such as steroids. The majority of contributing centers performed TPE every other day using a continuous centrifugation process for five treatments over 10 days. Plasma volume exchanged was 1 to 1.25 with 5\% albumin to achieve a fluid balance of $100 \%$. Most patients tolerated the procedures without any significant complications. However, the most common adverse event that occurred was hypocalcemic reactions, also known as citrate toxicity. This tended to be the probable cause of patients' symptoms and was considered a mild adverse reaction by the patients themselves. These reactions were minor and resolved with administration of calcium gluconate or carbonate. Overall, only $11.7 \%$ of patients had an adverse event, which was fewer than in a previous study with $23 \%$ of patients experiencing adverse events. ${ }^{16}$

In our patients, there was a lag of approximately 3 years between diagnosis of the disease and treatment with TPE. Early diagnosis leads to the employment of appropriate interventions for NMO/NMOSD patients, as they respond differently to treatments than do MS patients. ${ }^{6}$ Despite the delayed use of TPE in our patient population, most patients experienced mild to moderate improvement after TPE, with maximal improvement of symptoms achieved by the fourth or fifth treatment. It appears that adults continued to have improvement during the entire course of the treatment series. This suggests that perhaps a longer course of treatment should be employed in adults. However, we are unable to generalize this change in practice as we are limited by the small number of patients who were treated with more than five procedures in our study. We did not identify clinical characteristics such as paraparesis, blindness, etc., that responded better or worse to TPE. Our results were different from a recent retrospective study of 60 patients with severe NMO that demonstrated that initiating TPE earlier was a 
predictor of improved clinical outcomes. ${ }^{10}$ However, our findings support those of other studies that showed that the timing of TPE initiation did not affect clinical response. , $^{314,19,20}$

Our study demonstrated that patients between the ages of 22 and 50 had better CSI than children. The improvement was noted as "mild" to "moderate," for which nearly half of the patients did not receive additional TPE series. With regards to CSI, several studies found that TPE produced moderate to significant clinical improvement in patients. ${ }^{14,18}$ When reviewing the impact of the presence of antibody on treatment, we found no role of AQ4-seropositivity in predicting relapse or CSI. No other study to date has determined whether TPE affects relapse in seropositive patients. In addition, while moderate to significant CSI changes were seen in a study performed on seropositive patients, it is impossible to determine if seronegative patients would have had similar results, as none were included. ${ }^{14}$

Overall, there is a paucity of published clinical data regarding TPE in NMO/NMOSD patients. Although this study is a retrospective observational study, it represents the first large multicenter approach to assess the effects of TPE in this patient population. The limitations of this study include its retrospective design. Given that this study involved a retrospective medical chart review, it was limited by incomplete documentation, as information found in medical charts was often difficult to read or interpret. In addition, there was variability in the quality of medical information present in the patient charts. Future studies should include a prospective multicenter trial to formalize a treatment protocol with clearly defined outcome measures to determine the effect of TPE timing on clinical outcomes and to determine if patients would benefit from chronic TPE to prevent progression or relapse of NMO/NMOSD.

\section{I CONCLUSIONS}

TPE improved the CSI of patients, especially adults between the ages of 22 to 50 years-of-age. TPE was performed similarly at all the institutions in this study. Overall, TPE was safe and tolerable, with low adverse events.

\section{ACKNOWLEDGMENT}

ECCW is an employee of Quest Diagnostics.

\section{ORCID}

Tina S. Ipe (1D https://orcid.org/0000-0003-3554-5521 Jennifer Webb $\mathbb{D}$ https://orcid.org/0000-0003-1463-4963 Yanyun Wu (D) https://orcid.org/0000-0001-8410-4349 Chisa Yamada (10 https://orcid.org/0000-0002-1968-2385

\section{REFERENCES}

1. Lennon VA, Wingerchuk DM, Kryzer TJ, et al. A serum autoantibody marker of neuromyelitis optica: distinction from multiple sclerosis. Lancet. 2004;364(9451):2106-2112.

2. Takahashi T, Fujihara K, Nakashima I, et al. Anti-aquaporin-4 antibody is involved in the pathogenesis of NMO: a study on antibody titre. Brain. 2007;130(pt 5):1235-1243.

3. Meca-Lallana JE, Hernandez-Clares R, Leon-Hernandez A, Genoves Aleixandre A, Cacho Perez M, Martin-Fernandez JJ. Plasma exchange for steroid-refractory relapses in multiple sclerosis: an observational, MRI pilot study. Clin Ther. 2013;35(4): 474-485.

4. Mealy MA, Wingerchuk DM, Greenberg BM, Levy M. Epidemiology of neuromyelitis optica in the United States: a multicenter analysis. Arch Neurol. 2012;69(9):1176-1180.

5. Matiello M, Kim HJ, Kim W, et al. Familial neuromyelitis optica. Neurology. 2010;75(4):310-315.

6. Drori T, Chapman J. Diagnosis and classification of neuromyelitis optica (Devic's syndrome). Autoimmun Rev. 2014;13 (4-5):531-533.

7. Bonnan M, Cabre P. Plasma exchange in severe attacks of neuromyelitis optica. Mult Scler Int. 2012;2012:787630.

8. Padmanabhan A, Connelly-Smith L, Aqui N, et al. Guidelines on the use of therapeutic apheresis in clinical practice - evidencebased approach from the Writing Committee of the American Society for apheresis: the eighth special issue. J Clin Apher. 2019; 34(3):171-354.

9. Lehmann HC, Hartung HP, Hetzel GR, Stuve O, Kieseier BC. Plasma exchange in neuroimmunological disorders. Part 1. Rationale and treatment of inflammatory central nervous system disorders. Arch Neurol. 2006;63(7):930-935.

10. Bonnan M, Valentino R, Debeugny S, et al. Short delay to initiate plasma exchange is the strongest predictor of outcome in severe attacks of NMO spectrum disorders. J Neurol Neurosurg Psychiatry. 2018;89(4):346-351.

11. Wingerchuk DM, Lennon VA, Pittock SJ, Lucchinetti CF, Weinshenker BG. Revised diagnostic criteria for neuromyelitis optica. Neurology. 2006;66(10):1485-1489.

12. Wingerchuk DM, Banwell B, Bennett JL, et al. International consensus diagnostic criteria for neuromyelitis optica spectrum disorders. Neurology. 2015;85(2):177-189.

13. Keegan M, Pineda AA, McClelland RL, Darby CH, Rodriguez M, Weinshenker BG. Plasma exchange for severe attacks of CNS demyelination: predictors of response. Neurology. 2002;58(1): 143-146.

14. Kim SH, Kim W, Huh SY, Lee KY, Jung IJ, Kim HJ. Clinical efficacy of plasmapheresis in patients with neuromyelitis optica spectrum disorder and effects on circulating anti-aquaporin-4 antibody levels. J Clin Neurol. 2013;9(1):36-42.

15. Kleiter I, Gahlen A, Borisow N, et al. Neuromyelitis optica: evaluation of 871 attacks and 1,153 treatment courses. Ann Neurol. 2016;79(2):206-216.

16. Morgan SM, Zantek ND, Carpenter AF. Therapeutic plasma exchange in neuromyelitis optica: a case series. J Clin Apher. 2014;29(3):171-177.

17. Wang KC, Wang SJ, Lee CL, Chen SY, Tsai CP. The rescue effect of plasma exchange for neuromyelitis optica. J Clin Neurosci. 2011;18 (1):43-46 
18. Watanabe S, Nakashima I, Misu T, et al. Therapeutic efficacy of plasma exchange in NMO-IgG-positive patients with neuromyelitis optica. Mult Scler. 2007;13(1):128-132.

19. Magana SM, Keegan BM, Weinshenker BG, et al. Beneficial plasma exchange response in central nervous system inflammatory demyelination. Arch Neurol. 2011;68(7):870-878.

20. Weinshenker BG, O'Brien PC, Petterson TM, et al. A randomized trial of plasma exchange in acute central nervous system inflammatory demyelinating disease. Ann Neurol. 1999;46(6):878-886.
How to cite this article: Ipe TS, Raval JS, Fernando LP, et al. Therapeutic plasma exchange for neuromyelitis optica spectrum disorder: A multicenter retrospective study by the ASFA neurologic diseases subcommittee. J Clin Apher. 2020;35:25-32. https:// doi.org/10.1002/jca.21754 